\title{
Bipedal robot locomotion modelling with virtual height inverted pendulum and preview control approaches in Simulink environment
}

\author{
Ramil Khusainov', Ilya Afanasyev', Leysan Sabirova', Evgeni Magid ${ }^{2}$ \\ ${ }^{I}$ Intelligent Robotic Systems Laboratory, Innopolis University, \\ Innopolis City, Russian Federation \\ E-mail: \{r.khusainov, i.afanasyev, l.sabirova\}@innopolis.ru \\ ${ }^{2}$ Intelligent Robotic Systems Laboratory, Kazan Federal University, \\ Kazan, Russian Federation \\ E-mail:dr.e.magid@ieee.org
}

\begin{abstract}
Control algorithms development for humanoid locomotion is difficult due to humanoid's high number of Degrees of Freedom and system nonlinearity. Moreover, a humanoid may lose balance as its walking speed changes. We present AR-601M humanoid locomotion modelling with virtual height inverted pendulum and preview control approaches. A step length and period influence on walking stability were investigated and simulated within Simulink environment. Achievable in simulation maximal torques in leg joints and corresponding trajectories were verified against attainable robot motors' values.
\end{abstract}

Keywords: Biped robot, dynamic stability, Simulink, AR-601M robot

\section{Introduction}

Bipedal robot design and locomotion control are often referred to as extremely demanded and challenging research topics as stable locomotion is a minimal necessary requirement for all anthropomorphic robot applications. A number of successful dynamically stable biped locomotion approaches have been proposed over the last decades ${ }^{1,2,3,4,5,6}$, and a large number of them address walking stability issue with analytical methods that are based on locomotion dynamics and employ particular stability constraints. Among the most common stability constraints is a so-called Zero Moment Point (ZMP) approach ${ }^{7}$. ZMP criteria is applied for locomotion reference trajectory generation together with a significantly simplified robot model, for example, such as a Linear Inverted Pendulum Model $(\text { LIPM })^{8}$. Yet, as LIPM model does not count for full body dynamics, this causes ZMP errors, which in turn may become critical for a particular walking speed. To deal with this issue, $\mathrm{Ha}$ and $\mathrm{Cho}^{9}$ proposed a novel simplified robot model, which is called Virtual Height Inverted Pendulum Model (VHIPM) and experimentally demonstrated that the ZMP error could be significantly reduced by varying the height of Center of Mass $(\mathrm{CoM})$ in their LIPM model.

In this paper we present two pattern generation principles: preview control method and VHIPM method with LIPM model ${ }^{10,11}$. The algorithms modelling, experiments and testing were carried out in MATLAB/ Simulink environment. For VHIPM we calculated maximal speed of AR-601M stable walking and corresponding torques in the robot joints. The rest of the paper is organized as following. Section 2 presents the robot and its modelling. Section 3 describes walking trajectory generation with VHIPM method (with LIPM) 
and preview control method, followed by simulation results in Section 4. Finally, we conclude in Section 5.

\section{System setup}

\subsection{AR-601M robot description}

Figure 1 presents a developed by Russian company Android Technics AR-601M biped robot and our 41 degrees of freedom (DoF) AR-601M's modelling in MATLAB/Simulink environment. However, only 6 joints per each leg are active during locomotion (12 DoF in total): three joint axes are in the hip, two joints are at the ankle and one in the knee. Mass and size parameters of the robot legs are given in Table 1 . The total mass of the robot is $65 \mathrm{~kg}$ and the height is 1442 $\mathrm{mm}$; for further details about AR-601M the interested reader could refer to Khusainov et $\mathrm{al}^{12}$.

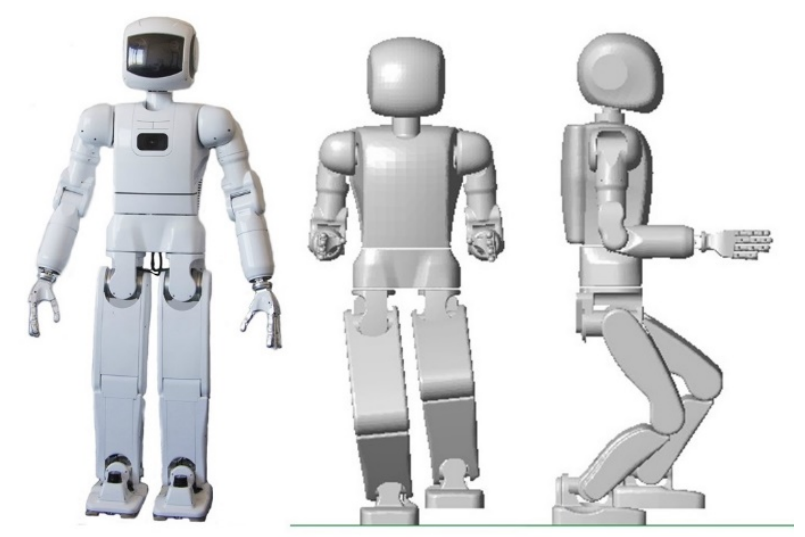

Fig. 1. Anthropomorphic robot AR-601M: photograph (left) and MATLAB/Simulink model (middle and right).

Table 1. Mass and size parameters of AR-601M legs.

\begin{tabular}{|l|l|l|}
\hline Link & Size parameters $(\mathrm{mm})$ & Mass $(\mathrm{kg})$ \\
\hline Thigh & Length $: 280$ & 7.5 \\
\hline Shank & Length $: 280$ & 6.9 \\
\hline Foot & LWH: $254 \times 160 \times 106$ & 3.2 \\
\hline
\end{tabular}

\subsection{Modelling in Simulink environment}

Initial solid CAD-generated AR-601M robot model in SolidWorks contained 59 parts and their linear dimensions corresponded to the real robot with a rather high accuracy. When we imported the robot model from CAD package, its mass distribution was considered as homogeneous, which is not the same as in the real robot because of heterogeneity of the robot parts (for example, the robot hand contains metal parts, printed circuit boards, actuators, wiring harness, etc., and all these components have different weight, unevenly distributed across the hand). Therefore, mass characteristics of the real robot (mass, CoM positions and moments of inertia) for each part were requested from the manufacturer and further integrated into the virtual environment model.

Link Software tool in Simulink SimMechanics library was used to generate a mechanical model of the robot in MATLAB SimMechanics environment automatically by importing its solid body CAD model from SolidWorks. We modelled each robot joint as a revolute joint with a single DoF. Figure 2 demonstrates a block diagram of AR-601M leg simulated in SimMechanics. The figure shows that the leg has 6 links and 6 rotational joints. The robot initial position is determined so that the legs are fully stretched out, the feet are parallel to the horizontal plane, and the choice of the hands' initial position is arbitrary.

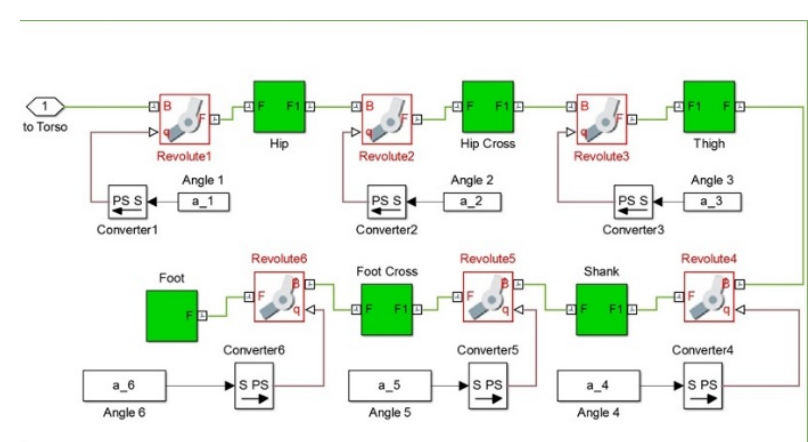

Fig. 2. A block diagram of AR-601M's leg, modelled in MATLAB/SimMechanics Toolbox.

A complete 3D simulation of multibody dynamics was performed using variable step period implicit solver (ODE 23t*) of MATLAB SimMechanics Toolbox. We modelled the robot feet ground contact with nonlinear spring-damper method ${ }^{13}$, where normal and friction forces are calculated according to Eq.1 and Eq.2 respectively:

$$
\begin{aligned}
& F_{n}=k_{n} z+b_{n} z \dot{z} \\
& F_{t}=b_{t} z \dot{x}, \quad F_{t} \leq \mu F_{n}
\end{aligned}
$$


where $k_{n}, b_{n}, b_{t}$ and $\mu$ are contact force parameters. The contact model details can be found in the previous work of the authors ${ }^{12}$.

\section{Walking Trajectory Generation}

\subsection{Virtual height inverted pendulum approach}

Bipedal robot locomotion could be viewed as a sequence of single steps ${ }^{14}$. Next, walking trajectory is calculated under the set of widely applied in biped walking experimental approaches' assumptions ${ }^{15}$ :

- A swing foot is always parallel to the ground plane (support plane) ${ }^{16}$

- An upper body always stays upright

- CoM of a robot is moving at a constant height

- A swing foot moves within a cycloid trajectory and its coordinates are described with the following equations:

$$
\begin{aligned}
& x(t)=x_{0} \\
& z(t)=-S \cos \left(\frac{\pi t}{T}\right) \\
& y(t)=0.5 H\left(1-\cos \left(\frac{2 \pi t}{T}\right)\right)
\end{aligned}
$$

where $x_{0}$ is a distance between two feet in $x$-direction, $S$ is a single step length, $H$ is a step height, $T$ is a step period. Yonemura et al. ${ }^{15}$ demonstrated that equations for CoM trajectory could be expressed as:

$$
\begin{aligned}
& \ddot{x}_{C O M}-\frac{g}{\alpha z_{C o M}} x_{C O M}=0 \\
& \ddot{y}_{C O M}-\frac{g}{\beta z_{C O M}} y_{C O M}=0
\end{aligned}
$$

where coefficients $\alpha$ and $\beta$ are obtained experimentally while minimizing ZMP error. Therefore, robot's CoM point trajectories are defined with the following expressions:

$$
\begin{aligned}
& x(t)=C_{1} e^{-w_{1} t}+C_{2} e^{w_{1} t}, w_{1}=\sqrt{\frac{g}{\alpha z_{C o M}}} \\
& y(t)=D_{1} e^{-w_{2} t}+D_{2} e^{w_{2} t}, w_{2}=\sqrt{\frac{g}{\beta z_{C o M}}}
\end{aligned}
$$

where $C_{l}, C_{2}, D_{1}$ and $D_{2}$ coefficients are found from initial and final values of CoM coordinates. After we define translational and rotational coordinates for the body and the swing foot, the joint angle trajectories are obtained applying inverse kinematics. Such trajectory assumes that the robot has some initial velocity. To move the robot from a resting state we initialize step length with a very small but reasonable value (i.e., a short step) and then gradually increase it in a loop to the maximal value until accumulated ZMP errors become critical and the robot finally falls down ${ }^{17}$.

\subsection{Preview control method}

For our virtual robot model a coordinate of ZMP was perceived as a feedback signal. Using a controller with the feedback allows the robot to adapt to a current situation and to calculate a necessary trajectory. Katayama et al. ${ }^{18}$ presented a preview control approach that uses a desired future value of the ZMP coordinates. We applied the same approach in order to obtain robot trajectories in frontal and sagittal planes. Thus, the ZMP coordinates for the inverted pendulum model (IPM) are given with the equation:

$$
P=X-\frac{Z_{c}}{g} \ddot{X}
$$

where $Z_{C o M}$ is the height of CoM, $g$ is the free fall acceleration and $X$ is the $x$-coordinate of CoM. Equations for the controller in a discrete time space have the following form:

$$
x(k+1)=A x(k)+B u(k), p(k)=C x(k)
$$

where $k$ is discrete time, $x(k)$ is a value of a state vector (position, velocity, acceleration of $\mathrm{CoM}), u(k)$ is a control vector, $p(k)$ is a value of ZMP coordinates. Coefficients $A, B, C$ are obtained from Eq.10, and expansion of the state vector $x$ in Taylor series to find its value in the next (discrete) instance of time ${ }^{8}$. A third derivative of $x$-coordinate $p(k)=\dddot{x}$ plays a role of control vector $u(k)$. According to Katayama et al. ${ }^{18}$, the control signal $u$ would apply a future value of the desired ZMP position and is calculated as follows:

$u(k)=-G_{I} \sum_{i=0}^{k} e(i)-G_{x} x(k)-\sum_{l=1}^{N_{l}} G_{d}(l) p_{d}(k+l)$

where $G_{I}, G_{x}, G_{d}$ are proportion coefficients, $e(i)$ is an error between calculated and desired ZMP coordinates, $p_{d}$ is the desired ZMP coordinates at $(k+1)$ time instance. Thus, the first term expresses an accumulated error between the calculated and the desired ZMP coordinates. The second term is proportional to a current state vector $x$. The third term takes into account 
future values of the desired ZMP position with the sum running over $N_{l}$ future values.

\section{Simulation results}

MATLAB/Simulink environment was used to model AR-601M walking and to test the algorithms. Table 2 presents walking trajectory parameters for experiments.

Table 2. Simulation parameters

\begin{tabular}{|l|l|}
\hline Parameter & Value \\
\hline Step height & $0.1 \mathrm{~m}$ \\
\hline Step period & $0.5 \mathrm{sec}$ \\
\hline CoM height & $0.75 \mathrm{~m}$ \\
\hline Step length & $0.1 \mathrm{~m}-$ max. value \\
\hline
\end{tabular}

\subsection{Virtual height inverted pendulum method}

First, maximum speed of the robot model, which could be achieved with linear inverted pendulum model (LIPM) method, was evaluated. A successful sequence of 100 robot steps is defined as a successful walking trial. After each successful walking trial, the step length

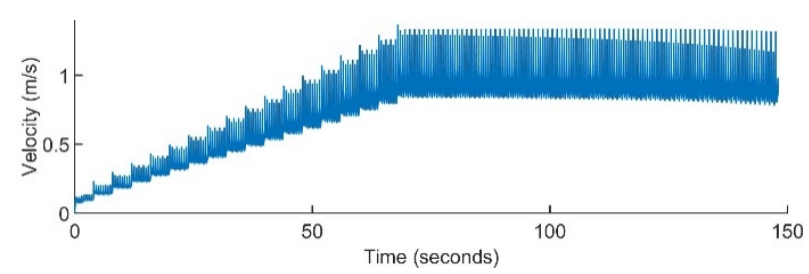

Fig. 3. Robot velocity in LIPM method.

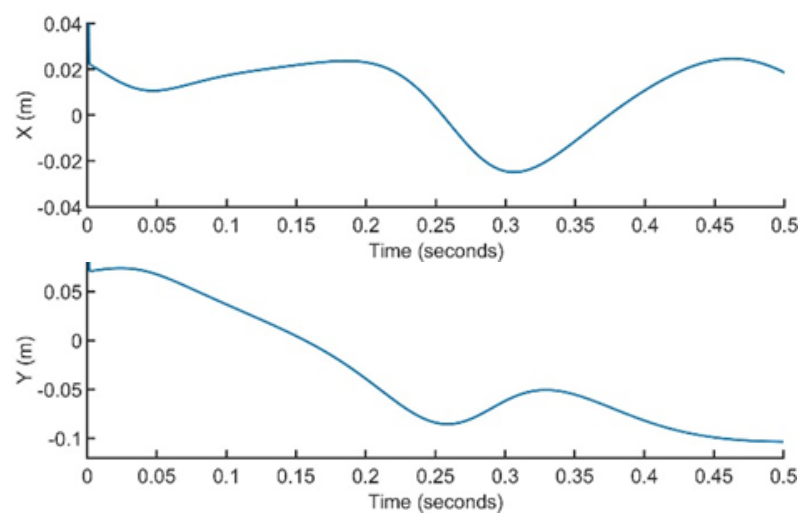

Fig. 4. ZMP $x$ (top) and $y$ (bottom) coordinates. was increased by $0.05 \mathrm{~m}$ and the algorithm was run again. The process of the step increase was repeated until the robot failed to execute a successful (stable) walking trial.

Simulations demonstrated that maximum step length at which the robot could perform stable walking is limited by $0.95 \mathrm{~m}$, which corresponds to $0.95 \mathrm{~m} / \mathrm{s}$ walking speed. CoM forward velocity value is shown in Fig.3. Figure 4 demonstrates ZMP coordinates for a step of $0.95 \mathrm{~m}$ length. A support foot center corresponds to $(0,0)$ coordinate. According to the calculated values, we have approximately $2.5 \mathrm{~cm}$ error for $x$-coordinate and $10 \mathrm{~cm}$ error for $y$-coordinate - just after a single step!

Next, we vary $\alpha$ and $\beta$ coefficients in Eq. 6 and 7 around 1 in order to minimize ZMP errors. Figure 5 shows the dependence of maximal error of ZMP coordinate on $\alpha$ for $x$-coordinate and on $\beta$ for $y$ coordinate. It was empirically detected that for $x$ coordinate the optimal value of coefficient $\alpha$ is equal to 1 , for $y$-coordinate optimal $\beta$ should lie within $[0.7,0.8]$ interval. Walking simulation with these coefficients showed that such optimization of trajectories allows to increase maximal step length from $0.95 \mathrm{~m}$ to $1.1 \mathrm{~m}$ and to achieve a maximal speed of $1.1 \mathrm{~m} / \mathrm{s}$ accordingly while keeping stable walking for at least 100 sequential steps.
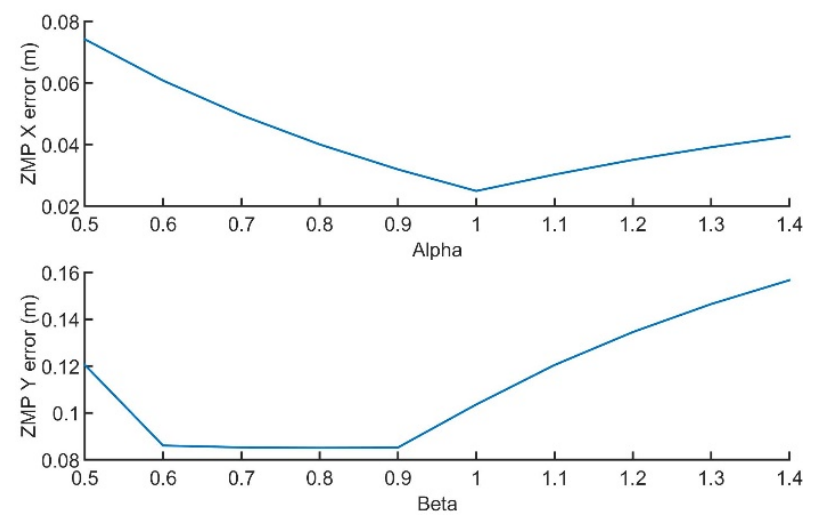

Fig. 5. Dependence of ZMP $x$-coordinate error on $\alpha$ (top) and $y$-coordinate error on $\beta$ (bottom).

Finally, we estimated the peak torque values in the ankle and knee joints that the robot motors should generate in order to support the locomotion according to the calculated trajectory. Figure 6 shows the calculated torque values for the ankle and the knee joints. 
The peak torque should approximately reach 100 $\mathrm{N}^{*} \mathrm{~m}$ for the ankle and $80 \mathrm{~N}^{*} \mathrm{~m}$ for the knee joint. According to the technical specifications of real AR601-M robot, its motors can generate maximal values of $50 \mathrm{~N}^{*} \mathrm{~m}$ for the knee and $20 \mathrm{~N} * \mathrm{~m}$ for the ankle joint. Unfortunately, this means that the calculated above maximal speeds could not be achieved for the real robot. Therefore, we are limited to use trajectories with a lower maximum speed, which are dynamically stable according to our simulations.
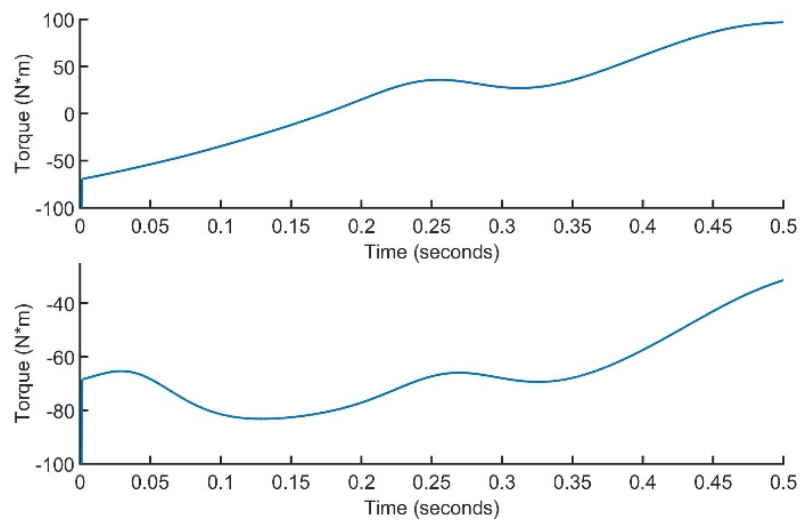

Fig. 6. Torque values in ankle joint (top) and knee joint (bottom).

\subsection{Preview control method}

In contrast to virtual height inverted pendulum approach, preview control method considers feedback of a ZMP error. Therefore, there is no need to make 100 steps (as in VHIPM method) in order to conclude if the walking is stable. If certain stable movement is achieved, robot will not fall down later under condition that there is no external disturbance. CoM position, desired and calculated ZMP coordinates for $\mathrm{x}$ and $\mathrm{y}$ coordinates are presented in Figures 7, 8. Figure 7 demonstrates that the calculated ZMP $x$-coordinate values are close to desired ZMP coordinate within acceptable error. The $x$-coordinate of CoM smoothly increases, which corresponds to the forward locomotion of the robot. Deviation of the calculated ZMP $y$ coordinate from desired values is shown in Figure 8, and its maximum is approximately $0,05 \mathrm{~m}$. Therefore, ZMP point is getting close to support polygon edge for a given walking speed and further increase of locomotion speed will result in robot downfall.

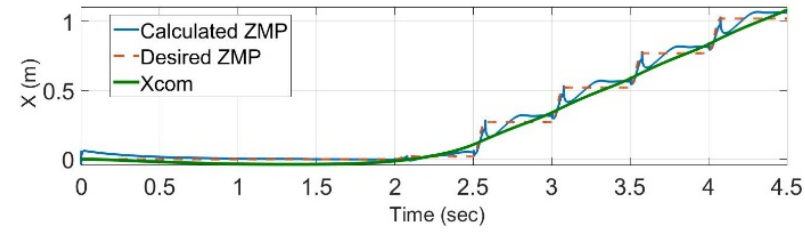

Fig. 7. Desired ZMP, calculated ZMP and CoM $x$-coordinate.

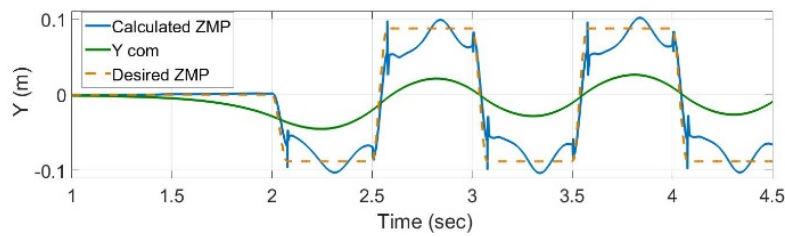

Fig. 8. Desired ZMP, calculated ZMP and CoM y-coordinate.

\section{Conclusions and Future work}

Our long-term research goal targets to develop and implement algorithms for dynamically stable locomotion of AR-601M robot. At the current stage of our research we modelled dynamically stable AR-601M robot locomotion with virtual height inverted pendulum model (VHIPM) and preview control methods. Experiments with simulated robot model were performed in MATLAB/Simulink environment.

VHIPM method allows to calculate a stable locomotion path quickly and effectively. We investigated in depth the influence of a step length and a step period on AR-601M's walking stability. In general, proper coefficients selection with a help of simulation for each particular robot model affords to achieve maximal walking speed for stable locomotion. The resulting torque values in the knee and the ankle joints could be calculated for such maximal walking speed and further verified against the technical capabilities of the real robot joint motors. Unfortunately, in our case the maximal speed of stable locomotion that was successfully demonstrated in the flat surface walking simulation turned out to be infeasible for AR601-M robot due to the limited capabilities of its joint motors. However, the knowledge of the theoretically possible speed within a simulation and the robot motors specifications give a good hint for the real achievable speed. As a part of our future work, we are going to implement the presented in this paper algorithms into 
AR-601M robot control system and verify that it could perform stable locomotion and achieve the calculated through simulations peak walking speed, which do take into account hardware limitations of the motors. The main weakness of this algorithm is the lack of feedback that results in accumulation of errors with time and which eventually may cause robot's downfall.

Preview control method does not have this disadvantage. However cart-table model doesn't take into account full dynamics of the robot motion, which leads to high ZMP errors at high walking speed. Therefore, as a part of our future work we schedule to consider full dynamics of the robot with influence of the upper body and swing leg motion and change mathematical model of locomotion dynamics accordingly.

\section{Acknowledgements}

This research has been supported by Russian Ministry of Education and Science as a part of Scientific and Technological Research and Development Program of Russian Federation for 2014-2020 years (agreement 14.609.21.0004, research grant ID RFMEFI60914X0004) and by Android Technics company, the industrial partner of the research.

\section{References}

1. J. Wright and I. Jordanov, Intelligent approaches in locomotion, International Joint Conference on Neural networks, (2012), pp. 1-8.

2. J. Pratt, C. M. Chew, A. Torres, P. Dilworth and G. Pratt, Virtual model control: An intuitive approach for bipedal locomotion, The International Journal of Robotics Research, 20 (2) (2001), pp. 129-143.

3. K. Lffler, M. Gienger and F. Pfeiffer, Sensor and control design of a dynamically stable biped robot, IEEE International Conference on Robotics and Automation, 1 (2003), pp. 484-490.

4. Y. Hurmuzlu, F. Gnot and B. Brogliato, Modeling, stability and control of biped robots a general frame- work, Automatica, 40 (10) (2012), pp. 1647-1664.

5. F. Asano and Z. W. Luo, Energy-efficient and highspeed dynamic biped locomotion based on principle of parametric excitation, IEEE Transactions on Robotics, $\mathbf{2 4}$ (6) (2008), pp. 1289-1301.

6. S. Jatsun, V. Dyshenko, A. Yatsun and A. Malchikov, Modelling of Robot's Motion by Use of Vibration of
Internal Masses, In Proceedings of EUCOMES 08, Springer Netherlands, (2009), pp.263-270.

7. M. Vukobratovich and B. Borovac, Zero-moment point thirty-five years of its life, International Journal of Humanoid Robotics, 1 (2004), pp.157-173.

8. S. Kajita, F. Kahehiro, K. Kaneko, K. Fujiwara, K. Harada, K. Yokoi and H. Hirukawa, Biped walking pattern generation using preview control of the zeromomentpoint, Proceedings of IEEE International Conference on Robotics and Automation, 2 (2003), pp. 1620-1626.

9. T. Ha and C.-H. Choi, An effective trajectory generation method for bipedal walking, Journal of Robotics and Autonomous Systems, 55 (10) (2007), pp.795-810.

10. R. Khusainov, I. Shimchik, I. Afanasyev and E. Magid, Toward a human-like locomotion: Modelling dynamically stable locomotion of an anthropomorphic robot in Simulink environment, Proceedings of the 12th International Conference on Informatics in Control, Automation and Robotics, 2 (2015), pp.141-148.

11. B. Gabbasov, I. Danilov, I. Afanasyev and E. Magid, Toward a human-like biped robot gait: Biomechanical analysis of human locomotion recorded by Kinect-based motion capture system. Proceedings of the 10th International Symposium on Mechatronics and its Applications, (2015), pp.1-6.

12. R. Khusainov, I. Shimchik, I. Afanasyev and E. Magid, 3D Modelling of Biped Robot Locomotion with Walking Primitives Approach in Simulink Environment. In Lecture Notes in Electrical Engineering, Springer International Publishing, 383 (30) (2016), pp. 287-304.

13. K.H Hunt. and F.R.E. Crossley, Coefficient of restitution interpreted as damping in vibroimpact, ASME Journal of Applied Mechanics, 42 (2) (1975), pp.440-445.

14. J. Denk and G. Schmidt, Synthesis of walking primitive databases for biped robots in 3-D environments, IEEE International Conference on Robotics and Automation, 1 (2003), pp.1343-1349.

15. A. Yonemura, Y. Nakajima, A.R. Hirakawa and A. Kawamura, Experimental approach for the biped walking robot MARI-1, 6th International Workshop on Advanced Motion Control, (2000), pp.548-553.

16. R. Khusainov, A. Klimchik and E. Magid, Swing leg trajectory optimization for a humanoid robot locomotion, Proceedings of the 13th International Conference on Informatics in Control, Automation and Robotics, 1 (2016), pp.130-144.

17. R. Khusainov, I. Shimchik, I. Afanasyev and E. Magid, Anthropomorphic robot modelling with virtual height inverted pendulum approach in Simulink: step length and period influence on walking stability. International Conference on Artificial Life and Robotics, (2016).

18. T. Katayama, T. Ohki, T. Inoue and T. Kato, Design of an optimal controller for a discrete-time system subject to previewable demand, International Journal of Control, 41 (3) (1985), pp.677-699. 